\title{
Multifocal Stereoscopic Projection Mapping
}
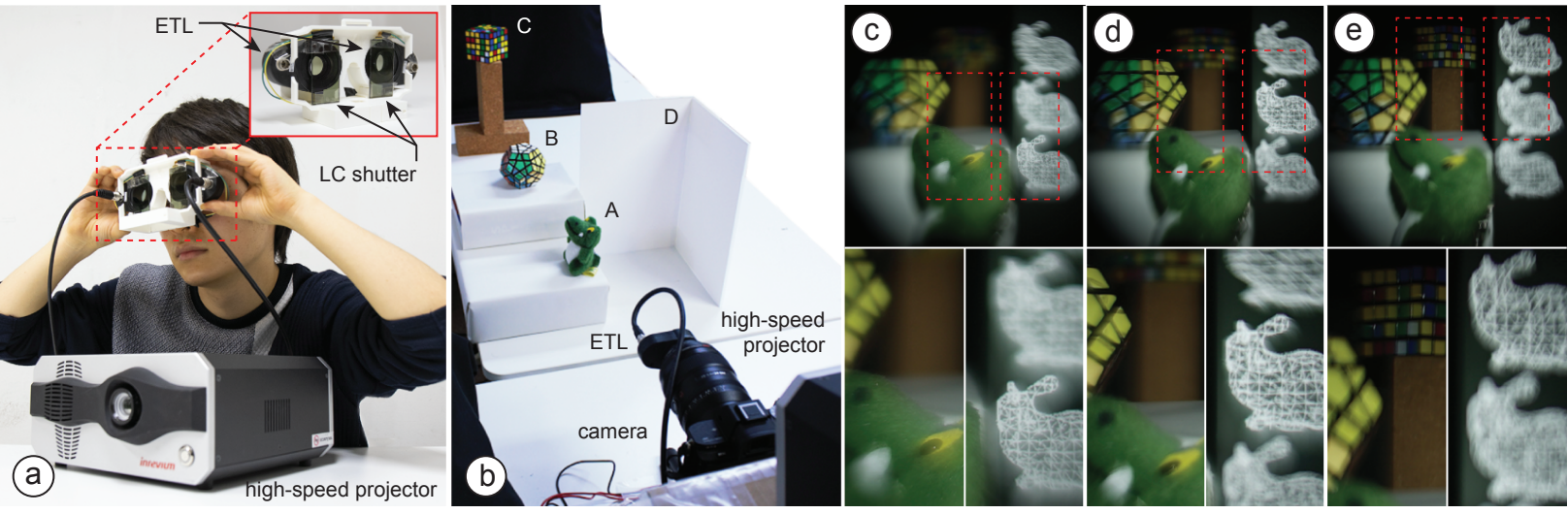

Fig. 1. The proposed multifocal stereoscopic projection mapping technique mitigates the vergence-accommodation conflict by placing the virtual image (as an optical term) at the desired distance from an observer using focal-sweep active shutter glasses and a synchronized high-speed projector. (a) Prototype system; (b) experimental setup to show the multifocal appearance of Stanford bunnies onto a flat surface (D) whose virtual images were placed, from near to far, at points at which three physical objects (A-C) were placed; (c-e) captured scene observed through a focal sweep lens. The bunnies, as well as the physical objects, appeared focused from the bottom to the top according to the focus distance of the camera lens at (c) near, (d) middle, and (e) far.

\begin{abstract}
Stereoscopic projection mapping (PM) allows a user to see a three-dimensional (3D) computer-generated (CG) object floating over physical surfaces of arbitrary shapes around us using projected imagery. However, the current stereoscopic PM technology only satisfies binocular cues and is not capable of providing correct focus cues, which causes a vergence-accommodation conflict (VAC). Therefore, we propose a multifocal approach to mitigate VAC in stereoscopic PM. Our primary technical contribution is to attach electrically focus-tunable lenses (ETLs) to active shutter glasses to control both vergence and accommodation. Specifically, we apply fast and periodical focal sweeps to the ETLs, which causes the "virtual image" (as an optical term) of a scene observed through the ETLs to move back and forth during each sweep period. A 3D CG object is projected from a synchronized high-speed projector only when the virtual image of the projected imagery is located at a desired distance. This provides an observer with the correct focus cues required. In this study, we solve three technical issues that are unique to stereoscopic PM: (1) The 3D CG object is displayed on non-planar and even moving surfaces; (2) the physical surfaces need to be shown without the focus modulation; (3) the shutter glasses additionally need to be synchronized with the ETLs and the projector. We also develop a novel compensation technique to deal with the "lens breathing" artifact that varies the retinal size of the virtual image through focal length modulation. Further, using a proof-of-concept prototype, we demonstrate that our technique can present the virtual image of a target 3D CG object at the correct depth. Finally, we validate the advantage provided by our technique by comparing it with conventional stereoscopic PM using a user study on a depth-matching task.
\end{abstract}

Index Terms-Stereoscopic projection mapping, multifocal display, vergence-accommodation conflict

\section{INTRODUCTION}

Stereoscopic projection mapping (PM) enables a user to see a threedimensional (3D) computer-generated (CG) object floating over the physical surfaces of arbitrary shapes around them using projected imagery. This technique can also be used to visually deform the surfaces. These illusory effects are achieved by tracking an observer's viewpoint, rendering the perspectively correct images with proper disparity for each eye, and projecting the two images in a time-sequential manner in each frame. The projected images are observed through active-shutter

\footnotetext{
- Sorashi Kimura, Daisuke Iwai, Parinya Punpongsanon, and Kosuke Sato are with the Graduate School of Engineering Science, Osaka University. E-mail: E-mail: see https://www.sens.sys.es.osaka-u.ac.jp/.

- Daisuke Iwai is with PRESTO, Japan Science and Technology Agency.
}

Manuscript received xx xxx. 201x; accepted xx xxx. 201x. Date of Publication xx xxx. 201x; date of current version xx xxx. 201x. For information on obtaining reprints of this article, please send e-mail to: reprints@ieee.org. Digital Object Identifier: $x x . x x x x / T V C G .201 x . x x x x x x x$ glasses consisting of liquid crystal (LC) shutters, which prevent interference between the two images for both eyes. There are several technical advantages of using stereoscopic PM over other types of augmented reality (AR) displays such as optical see-through (OST) and video see-through (VST) head-mounted displays (HMD). First, the field-of-view (FOV) can be made as wide as possible by increasing the number of projectors to cover the entire environment. Second, the active-shutter glasses used are normally much lighter, and, thus, their physical burden is less than HMDs. Third, multiple users can share the same AR experience if their viewpoints are sufficiently close to each other. Thanks to these advantages, researchers have found stereoscopic PM to be suitable for a wide range of applications, including but not limited to museum guides [63], architecture planning [8], product design [68], medical training [35], shape-changing interfaces [40], and teleconferencing [5].

In many of these applications, it is important to create a faithful impression of the 3D structure of the projected object or scene. However, like most commercial virtual reality (VR) and AR displays, current stereoscopic PM only satisfies binocular cues and is not capable of 
providing the correct focus cues, which causes a problem called the vergence-accommodation conflict (VAC). Vergence refers to the simultaneous movement of both eyes in opposite directions to obtain single binocular vision, while accommodation refers to the process by which each eye changes its optical power to focus on an object as its distance varies. The uncoupling of the vergence and accommodation causes significant discomfort, fatigue, and a distorted 3D perception for the observer [24]. While many approaches have been proposed to mitigate the VAC in VR/AR displays (see Sect.2), to the best of our knowledge, no technical solution has yet been presented for the VAC in stereoscopic PM.

We propose a multifocal approach to improve the mismatch between vergence and accommodation in stereoscopic PM. Our primary technical contribution is to attach electrically focus-tunable lenses (ETL) to active-shutter glasses to control both vergence and accommodation. Specifically, we apply fast and periodical focal sweeps to the ETLs, which causes the "virtual image" (as an optical term) of every part of the real scene seen through the ETLs to move back and forth during each sweep period. In each frame, we first render the perspectively correct images of a 3D CG object for both eyes. Then, we project them from a synchronized high-speed projector at the exact moment that the virtual image of the projected imagery on a real surface is located at a desired distance from the ETLs. Thus, each part of the surface is projected for a short period of time during each sweep period. The frequency of the focal sweep is higher than the critical flicker fusion frequency (CFF) so that the observer perceives the time integral of the scene appearances during the sweep period.

This paper introduces the technical details of our computational algorithms, which are jointly designed with the optics and hardware. Particularly, we solve three technical issues that are unique to stereoscopic PM: (1) The 3D CG object is displayed on non-planar and even moving surfaces; (2) the physical surfaces that are also seen through the ETLs need to be shown without the focus modulation; (3) the LC shutter glasses also need to be synchronized with the ETLs and the projector to provide the correct binocular cues as well as the focus cues. On the other hand, in other AR/VR displays, (1) the 3D CG object is displayed on a planar and fixed display panel, (2) observers do not see a real scene through ETLs, and (3) shutter glasses are not used We also develop a novel compensation technique to address the "lens breathing" artifact, which varies the retinal size of the virtual image through focal length modulation. Using a proof-of-concept prototype, we demonstrate that our technique can present the virtual image of a target 3D CG object with the perspectively correct appearance at the correct depth. Finally, we validate the advantage provided by our technique with regard to the mitigation of the VAC by comparing it with a conventional stereoscopic PM using a user study on a depth-matching task.

To summarize, our primary contributions are that we

- introduce a multifocal stereoscopic PM technique that mitigates the VAC by combining fast and periodical focal sweeps with active-shutter glasses and synchronizing high-speed projection,

- jointly design optics, hardware, and computational algorithms to present a 3D CG object with correct binocular and focus cues, solve three technical issues unique to stereoscopic PM, and propose a general computational solution for the lens breathing artifacts,

- implement a proof-of-concept prototype that displays an object's virtual image that is perspectively correct and located at a desired distance from the observer, and

- demonstrate the VAC mitigation achieved by the proposed system using a user study on a depth-matching task.

\section{Related Work}

\subsection{Stereoscopic PM}

Since the pioneering work conducted on rendering perspectively correct images on non-planar physical surfaces using projected imagery [59], much research has been carried out on stereoscopic PM. Due to several advantages, such as the wider FOV and fewer physical burdens than other types of VR/AR displays, stereoscopic PM has been applied in various fields. Telepresence has been considered a particularly suitable application of stereoscopic PM. The 3D shape and appearance of a distant person are captured using an RGB-D camera and displayed on local physical surfaces using projected imagery to make it appear as the distant person exists in the same room [6, 57| or behind a wall [5, 59]. Stereoscopic PM has also been applied in architecture planning [8 9], where new structures of a building are displayed on a wall of the building. In the car industry, it was found that stereoscopic PM has the potential to assist in interior designing such that designers can examine the shape of a dashboard without needing to fabricate physical mock-ups [68]. Stereoscopic PM has also been applied in medical training [35], where human organs inside a body are projected on the body. By focusing on expanding the application fields, previous works have not sufficiently considered the VAC, which, thus, remains an unsolvable and challenging technical issue in stereoscopic PM.

Various types of human perceptual properties in stereoscopic PM have been investigated. For example, Okutani et al. found that stereoscopic PM induces stereoscopic capture where the depth observed during stereoscopic PM is automatically attributed to the texture of a physical surface even when the texture conveys no disparity information [54]. Other researchers found that pseudo-haptic illusions occur in stereoscopic PM, where a user can feel bumps on a physically flat surface on which apparent bumps are overlaid [34]. Depth perception in stereoscopic PM has been more intensively analyzed than the other properties. Certain researchers tested the depth estimation ability of human participants given the binocular disparity in stereoscopic PM [21 62], while others did it with additional apparent cues such as color and texture [64]. However, due to the lack of a stereoscopic PM system that satisfies both binocular and focus cues, how the depth estimation accuracy is affected when the VAC is mitigated has not yet been investigated.

\subsection{Mitigating the VAC}

In VR and AR applications, CG images need to create a faithful impression of the $3 \mathrm{D}$ structure of the depicted object or scene. Unfortunately, commercially available 3D displays often yield perceptual distortions in the 3D structure due to the mismatch between binocular and focus cues. The VAC causes viewer fatigue and discomfort as well as distortions in the perceived depth [24, 72]. Much research has been carried out in an attempt to solve the VAC issue in both VR and AR displays, which include varifocal displays (VR [32 36 38 38 55] and AR [1 10 15 18 30 75]), multifocal displays

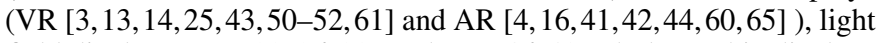
field displays (VR |23,26, 39] and AR [46, 48]), holographic displays (VR [11, 12 49, 58 and AR |30,47| ), and other display techniques, such as those that use color information [2 17]. Unfortunately, despite the substantial effort that has been put into solving the VAC for stereoscopic flat-panel displays and HMDs, researchers have not focused on the issue for stereoscopic PM. Previous works closely related to stereoscopic PM are associated with light field [23] and tomographic [31] projection systems. These technologies have been developed for conventional flat projection screens and, thus, are not directly applied to PM, where the projection target includes potentially non-planar and even moving surfaces. Furthermore, these works required customized, special projection devices, which potentially hinder developers from using them in the actual fields of application.

Among the four display technologies mentioned above, light field and holographic displays are not suitable for stereoscopic PM. Since it is not assumed to install tailored optical elements in the environment in general PM applications, light field and holographic displays-which, for instance, need to embed an angle-expanding material [23] and a phase modulator [47] onto physical surfaces - are not generally applicable for stereoscopic PM. Varifocal and multifocal displays basically share the same optical display architecture-both apply focus-tunable near-eye optics for displaying 3D CG objects with correct focus cues. The major difference between the two displays is that varifocal displays 

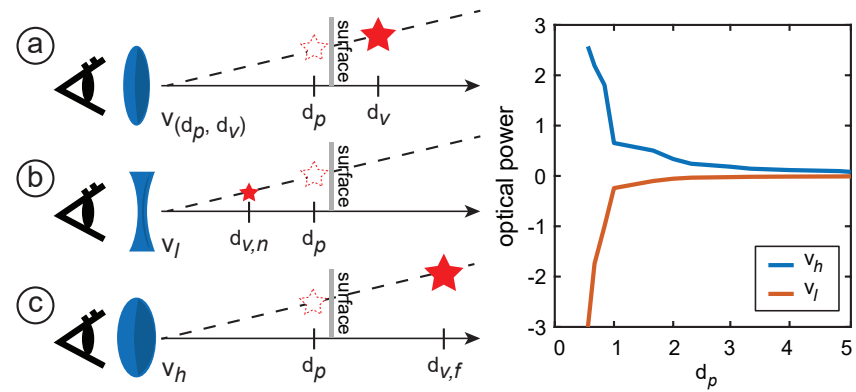

Fig. 2. (Left) The virtual image (solid star) of a projected object (dashed star) on a physical surface observed through an ETL. (a) The virtual image is located at $d_{v}$ from the ETL when the projected image is at $d_{p}$, and the ETL's optical power is $v\left(d_{p}, d_{v}\right)$. (b)(c) When the optical power is periodically swept between $v_{l}$ and $v_{h}$, the virtual image also periodically moves between the near end $d_{v, n}$ and the far end $d_{v, f}$. (Right) The required sweep range of an ETL's optical power when $d_{p}$ is changed under the condition that $d_{v, n}$ and $d_{v, f}$ are fixed at $\frac{1}{6} \mathrm{~m}$ and $\frac{1}{0.4} \mathrm{~m}$, respectively.

actively track the accommodation of the eye in real-time and provide a correct image at the interested depth, while multifocal displays passively offer multiple depths to simulate a 3D scene without considering where the eye focuses [78]. In stereoscopic PM, the real scene is also seen through the focus-tunable near-eye optics because a 3D CG object is projected directly onto a surface in the scene. Therefore, when a varifocal approach is employed, a user needs to observe the real scene with a focal setting that is optimized for the projected 3D CG object. This focus modulation can possibly create an unnatural view of the real scene. In addition, the projection surface can be non-planar. In this case, the projected 3D CG object is not positioned at the same distance from the observer, and, thus, a spatially-varying focal control is required to provide the correct focus cues. However, a varifocal approach assumes a spatially uniform operation. Furthermore, it is not preferable to add an eye-tracking sensor system to the active-shutter glasses because it degrades the advantage of stereoscopic PM (i.e., it only requires a lightweight eye wear) over the other VR/AR displays. Therefore, we adopt the multifocal approach in this research to mitigate the VAC in stereoscopic PM.

User studies have revealed that the observer's depth estimation performance can be significantly improved by mitigating the VAC in both VR [32 36 38, 55] and AR [66 67] displays. Therefore, this improvement could happen in stereoscopic PM as well. However, as mentioned in Sect.2.1 there is no experimental evidence of this due to the lack of a PM system satisfying both binocular and focus cues.

\subsection{Our contributions}

This paper presents the first stereoscopic PM system that mitigates the VAC by applying a multifocal approach. On top of the conventional stereoscopic PM framework, we apply additional optics (ETLs) and computational algorithms to achieve this goal. In particular, we describe how to solve the technical issues that are unique to stereoscopic PM, one of which is that the display surface is potentially non-planar and moving. An advantage of our technique is that it does not need customized, special projection devices and works with off-the-shelf projectors. We also conduct the first user study to investigate how the VAC mitigation improves the depth estimation accuracy in stereoscopic PM using a prototype system.

\section{Method}

\subsection{Virtual image of projected imagery seen through ETL}

We attach an ETL each to the left and right of LC shutter of the activeshutter glasses. As a result, a user can observe the virtual image of the projected imagery on a physical surface through the ETL. Suppose that the distance of the surface from the ETL is $d_{p}$ (Fig. 2a). Using the thin lens equation, we can determine the optical power $v\left(d_{p}, d_{v}\right)$ to locate the virtual image of a $3 \mathrm{D}$ CG object projected on the surface at a
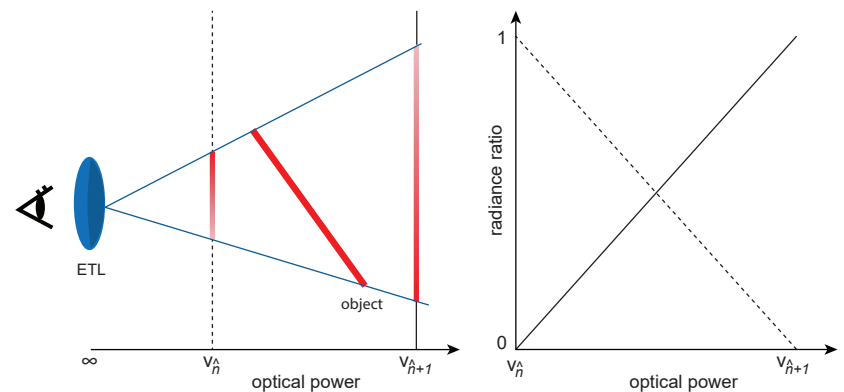

Fig. 3. Depth filtering technique. (Left) A depicted example showing the radiance distribution between the two nearest projector frames and (right) radiance distribution in linear proportion with distance measured in diopters.

desired distance $d_{v}$ from the ETL as follows:

$$
v\left(d_{p}, d_{v}\right)=\frac{1}{d_{p}}-\frac{1}{d_{v}} .
$$

The optical power is the reciprocal of the focal length $(\mathrm{m})$ and is generally represented in diopter (D). In this paper, we do not fix $d_{p}$ because the surface can be non-planar and can move in stereoscopic PM. Therefore, we place the virtual image of a projected 3D CG object at a desired distance by adjusting the optical power $v\left(d_{p}, d_{v}\right)$ according to both $d_{p}$ and $d_{v}$.

We apply a fast focal sweep to the ETL, where its optical power is periodically modulated between $v_{l}$ and $v_{h}\left(v_{l}<v_{h}\right)$ at $f \mathrm{~Hz}$ such that $f$ is higher than the CFF. When the physical surface is lit by normal room lighting, the virtual image of the surface periodically moves between the near end (when the optical power is $v_{l}$ ) and the far end (when it is $v_{h}$ ) along the ETL's optical axis. In our system, we turn off the room light and project the desired 3D CG object onto the surface when the optical power of the ETL becomes $v\left(d_{p}, d_{v}\right)$ for each focal sweep period. We can locate the virtual image at the desired distance $d_{v}$ under the condition that the optical power is within the range of $v_{l} \leq v\left(d_{p}, d_{v}\right) \leq v_{h}$.

Given the desired location range of the virtual image of a projected 3D CG object and the position of a projection surface, the sweep range required to place the virtual image at the desired location is determined. Suppose that the distance from the near end of the virtual image to the surface is $d_{v, n}$ and that from the far end to the surface is $d_{v, f}$ (Figs. 2p and 2 ), the relationship between $v_{l}$ and $d_{v, n}$ and that between $v_{h}$ and $d_{v, f}$ can be formulated as follows according to the thin lens equation:

$$
v_{l}=\frac{1}{d_{p}}-\frac{1}{d_{p}-d_{v, n}}, v_{h}=\frac{1}{d_{p}}-\frac{1}{d_{p}+d_{v, f}} .
$$

The right-hand side graph in Fig. 2 shows the required sweep range computed by Eq. 2 when $d_{p}$ is changed from $\frac{1}{3} \mathrm{~m}$ to $\frac{1}{0.2} \mathrm{~m}$ under the condition that $d_{v, n}$ and $d_{v, f}$ are fixed at $\frac{1}{6} \mathrm{~m}$ and $\frac{1}{0.4} \mathrm{~m}$, respectively. The graph indicates that the required sweep range becomes larger when the distance between the ETL and the surface is shorter. Therefore, we determine the sweep range from the desired location range of the virtual image of a 3D CG object projected onto the nearest surface from the ETL.

In an actual setup, the projector can display only a limited number of images $(N)$ during a sweep period. Therefore, it is not always possible to project a 3D CG object when the optical power of the ETL is exactly $v\left(d_{p}, d_{v}\right)$. To overcome this constraint, we sample $N^{\prime}(\leq N)$ optical powers $v_{n}\left(n=1, \cdots, N^{\prime}\right)$ within the range between $v_{l}$ and $v_{h}$ such that the projector can display an image at each $v_{n}$. Then, a 3D CG object is projected at the $\hat{n}$-th optical power in each sweep period when $v_{\hat{n}}$ is the closest to $v\left(d_{p}, d_{v}\right)$; thus,

$$
\hat{n}=\underset{n}{\arg \min }\left|v_{n}-v\left(d_{p}, d_{v}\right)\right| .
$$


The sampling of the optical power causes a discontinuity in the virtual image of a displayed 3D CG object when the optical power $v\left(d_{p}, d_{v}\right)$ is spatially varying and consequently spans two sampled optical powers (e.g., $v_{\hat{n}}$ and $v_{\hat{n}+1}$ ) (Fig. 3. To alleviate this discontinuity, we apply the "depth filtering" technique introduced in previous work [3]. Depth filtering distributes the radiance between the two nearest projector frames in linear proportion, with distance measured in diopters. Suppose $r_{\hat{n}}$ and $r_{\hat{n}+1}$ are the distributed radiance of the original radiance $r$ to the $\hat{n}$-th and $(\hat{n}+1)$-th projection images, respectively; they would be computed as follows:

$$
r_{\hat{n}}=r\left(1-\frac{v\left(d_{p}, d_{v}\right)-v_{\hat{n}}}{v_{\hat{n}+1}-v_{\hat{n}}}\right), r_{\hat{n}+1}=r-r_{\hat{n}} .
$$

A previous user study showed that depth filtering could provide continuous focus cues [45]. Another work recommended that the sampling interval of the optical power should be less than $0.6 \mathrm{D}$ to provide perceptually continuous focus cues [74]. We follow this guideline when we build our prototype.

\subsection{Synchronization}

To display the virtual image of a 3D CG object at the desired location, we need to synchronize the projector, ETLs, and shutter glasses. We determine our synchronization strategy while considering the following temporal response properties of the devices:

Projector: We employ a state-of-the-art off-the-shelf high-speed projector [73]. It projects an image at $\delta t_{p} \mathrm{~ms}$ after a trigger signal is sent from the PC. It is also known that the delay does not vary over time. Therefore, once we measure $\delta t_{p}$ in the calibration phase, we can project images at the desired timings by sending the triggers at $\delta t_{p}$ prior to the projection. There is also a delay when transferring an image from the PC to the projector $(\sim 3 \mathrm{~ms})$. We apply a multi-threading pipeline to perform the projection and transfer the images in parallel, and, thus, the delay of the latter process does not have to be considered during synchronization.

ETLs: We use polymer-based ETLs in which the lens consists of an optical fluid sealed off by an elastic polymer membrane. An electromagnetic actuator ring exerts pressure on the outer zone of the lens, which changes its curvature, i.e., the optical power. Polymer-based ETLs can achieve a faster focal change than other ETL types while maintaining a relatively large aperture size. As previous focal sweep systems [13 31 37, 60] have shown, the optical power of an ETL can be smoothly modulated when a sinusoidal or triangular wave is applied as the electrical current. Therefore, we apply a sinusoidal wave as the input in our system. It was also experimentally validated that the temporal response of an ETL is a linear time-invariant (LTI) system [28]. Therefore, when we supply a sinusoidal wave $i[\phi]$ to an ETL, the output optical power also becomes a periodical wave $v[\phi]$, where $\phi$ is the phase of the input wave signal $(0 \leq \phi<2 \pi)$. Once we measure one cycle of $v[\phi]$ in the calibration phase, we can estimate the optical power at any timing required.

Shutter glasses: We use LC-based active-shutter glasses. The LC is transparent (open) in the initial state and becomes opaque (closed) when a voltage is applied. There are two delays to be considered. First, a delay $\delta t_{o 2 c}$ occurs between the moment we apply the voltage to change the LC state from open to closed till the moment the LC shutter actually closes. Second, there is a longer delay $\delta t_{c 2 o}$ that occurs when it shifts from the closed to the open states as well. Therefore, we measure these delays during the calibration phase and use them for synchronization.

Figure 4 shows the timing chart of our synchronization strategy. First, the ETLs for the left and right eyes are modulated by supplying them with the same sinusoidal waves $i[\phi]$ of frequency $f \mathrm{~Hz}$. We divide the optical power waveform into two parts in each period and use the upward curve (from $v_{l}$ to $v_{h}$ ) for the left eye and the downward curve (from $v_{h}$ to $v_{l}$ ) for the right eye. The projector projects images

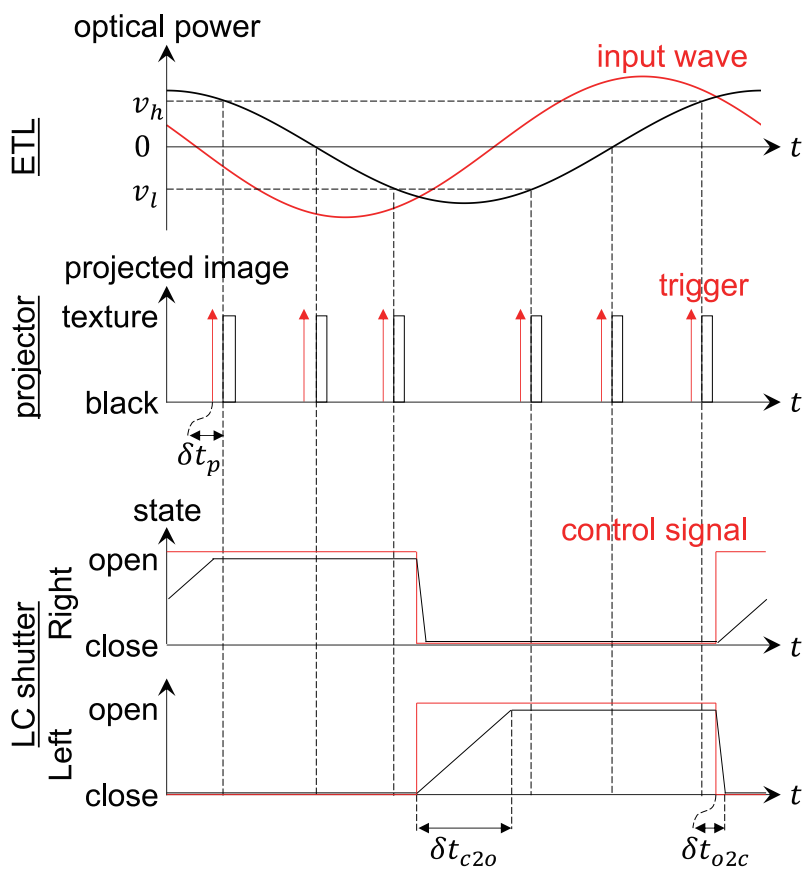

Fig. 4. Timing chart of the proposed synchronization strategy. In this example, the number of the sampled ETL's optical power is three (i.e., $N^{\prime}=3$ ), at which the high-speed projector projects images.

when the optical power $v[\phi]$ of each ETL becomes $v_{n}$. To make this happen, we send a trigger signal to the projector when the phase of the ETL's input signal is $2 \pi f \delta t_{p}$ prior to the target phase. We open the LC of the left eye and close the LC of the right when the ETLs' optical powers form the upward curves. In the same way, we open the LC of the right eye and close the LC of the left when the optical powers form the downward curves. We control the LC shutters such that the optical powers become equal to the maximum and minimum values during the closing/opening transition time of the LC shutters because the time derivative of the modulated optical power of each ETL becomes smallest at these timings.

Stereoscopic PM applications generally project a white color onto physical surfaces to make them visible in addition to the projected 3D CG object to enable AR experiences. In our system, the physical surfaces under white illumination are also observed through the ETL. To avoid a situation in which the locations of the virtual images of these surfaces vary according to the focal sweep, we illuminate them when the optical powers of the ETLs are zero (i.e., $v[\phi]=0$ ). This allows us to present the physical surfaces without any focus modulations.

\subsection{Lens breathing compensation}

The virtual image seen through the ETL is scaled using the change in its optical power because an observer's eye is not co-located with the ETL but, instead, placed behind it. When a projected 3D CG object is divided into multiple parts that are observed through the ETL with different optical powers, the lens breathing phenomenon causes an overlap or gap between their virtual images on the retina. This artifact has been noticed in previous works [31.38 52], in which it was solved by manually adjusting the size of displayed images. In this paper, we propose a simple yet general computational solution for this issue.

Without loss of generality, we suppose simple situations where a 3D CG object is projected onto a flat surface that is located $d_{p}$ away from an ETL (Figs. 5(a1) and 5(b1)). The object is divided into two parts that are observed with different optical powers. When the optical power becomes equal to a certain non-zero value, the inner part of the object with respect to the ETL's optical axis is projected onto the surface. When the optical power becomes zero, the outer part is projected. If the height of the inner part on the surface is $h_{p}$, then that of its virtual 

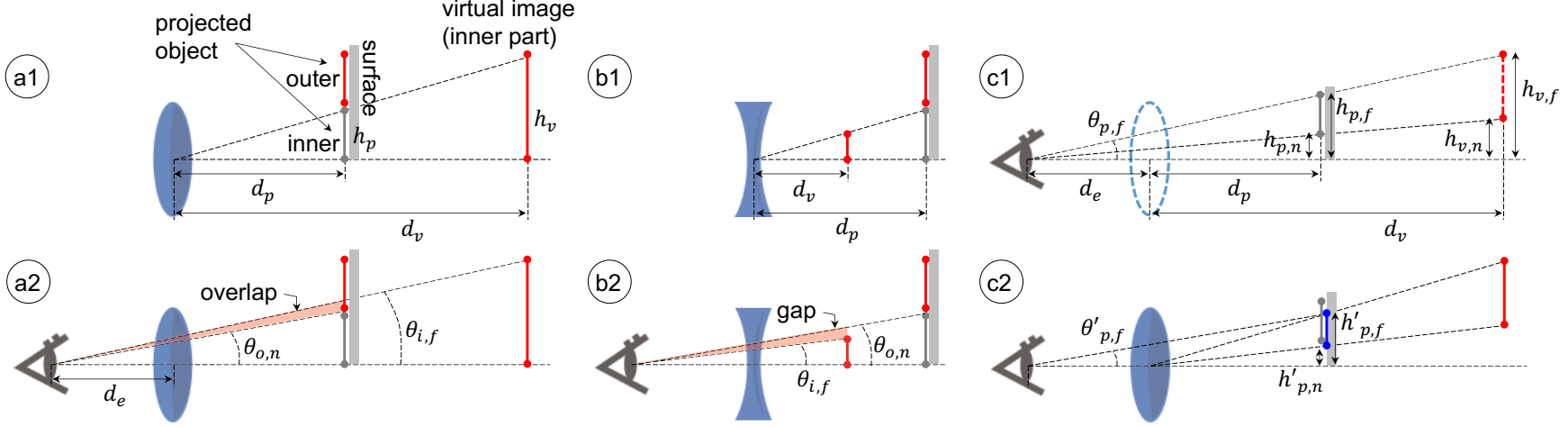

Fig. 5. Lens breathing artifact and compensation. When the inner part of a projected object is observed with a (a) positive or (b) negative optical power, an (a) overlap or (b) gap occurs between its virtual image and that of the outer part observed with the optical power of zero. Note that indicates an observed real image and the virtual image of - . (c) The proposed compensation technique resizes the projected object to -

image $h_{v}$ is computed as $h_{p} \frac{d_{v}}{d_{p}}$ according to geometric similarity, where $d_{v}$ is the distance from the ETL to the virtual image. Because the outer part is observed when the ETL's optical power is zero, its real image is observed. Now, we suppose that an observer's eye is located at $d_{e}$ behind the ETL (Figs. 5(a2) and 5(b2)). Regarding the virtual image of the inner part, we denote the visual angle of its far end from the optical axis as $\theta_{i, f}$. We also denote the visual angle of the near end of the outer part as $\theta_{o, n}$. Figures 5 (a2) and 5 (b2) depict the relationship between $\theta_{i, f}$ and $\theta_{o, n}$ for both cases where the non-zero optical power is positive and negative, respectively. In general, when a larger optical power is applied to the inner part of a 3D CG object than the outer part, $\theta_{i, f}>\theta_{o, n}$, and, consequently, the retinal images of the inner and outer parts overlap. On the contrary, when a smaller optical power is applied to the inner part, $\theta_{i, f}<\theta_{o, n}$, and a gap occurs between the two retinal images.

As discussed above, in a case where a 3D CG object is divided into multiple parts that are observed with different optical powers, an overlap or gap of their virtual images occurs on the observer's retina. We compensate for these artifacts by resizing each part of the projected object. The following method assumes that each part of the object is projected onto a planar surface that is perpendicular to the ETL's optical axis. If a part of the object that is observed with the same optical power covers a non-planar surface, we further divide it into smaller parts until each surface area onto which the subdivided part is projected can be regarded as a plane that is perpendicular to the optical axis. Suppose that $\theta_{p, n}$ is the visual angle of the near end of a part of a 3D CG object on the projection surface, and $\theta_{p, f}$ is that of its far end in a case where the object is not seen through an ETL (Fig. 5.c1)). Then, our method aims to resize the object so that the visual angles of the far and near ends of its virtual image correspond to $\theta_{p, n}$ and $\theta_{p, f}$, respectively. Let the height of the near end and that of the far end be $h_{p, n}$ and $h_{p, f}$, respectively. To achieve our goal, the heights of the near and far ends of its virtual image ( $h_{v, n}$ and $h_{v, f}$, respectively) should be $h_{v, *}=\frac{d_{v}+d_{e}}{d_{p}+d_{e}} h_{p, *}$ according to geometric similarity, where $*$ represents either $n$ or $f$. The ETL represents this virtual image as the projection of the displayed 3D CG object from the ETL's optical center onto a plane located at $d_{v}$ away from the ETL. Therefore, to show this virtual image, the heights of the near and far ends of the 3D CG object on the surface should be $h_{p, *}^{\prime}=\frac{d_{p}}{d_{v}} h_{v, *}$ according to geometric similarity (Fig. 5. (c2)). Consequently, we can show the virtual image of the 3D CG object such that the visual angles of its far and near ends correspond to $\theta_{p, *}$ by resizing the object using the following equation:

$$
h_{p, *}^{\prime}=\frac{d_{p}\left(d_{v}+d_{e}\right)}{d_{v}\left(d_{p}+d_{e}\right)} h_{p, *}
$$

An important consequence that is indicated by Eq. 5 is that the resizing factor is constant for the entire area of a subdivided part of the 3D CG

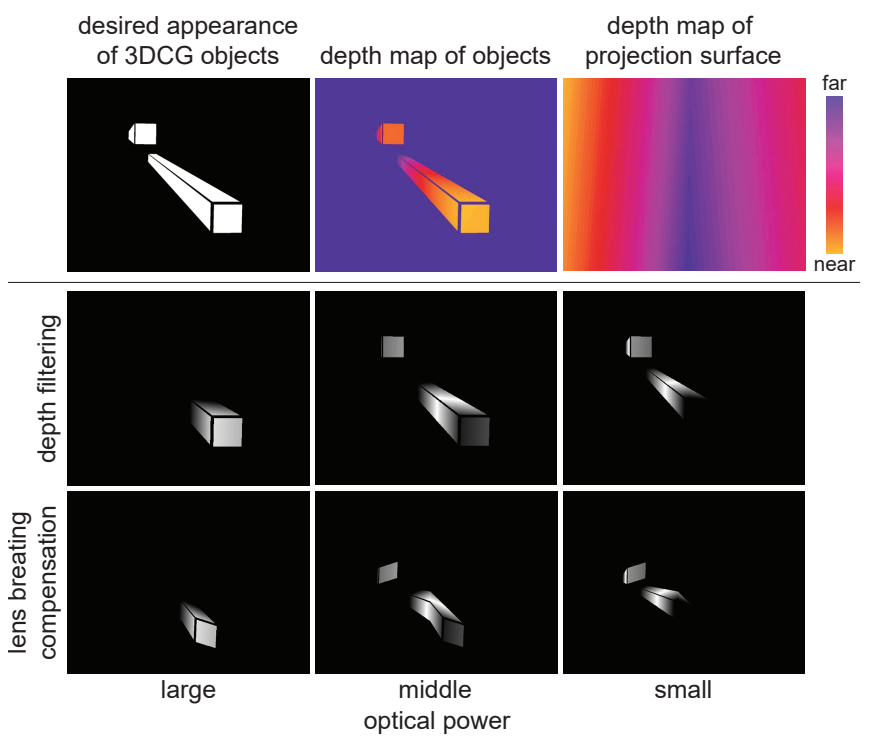

Fig. 6. Generated images in the proposed rendering pipeline, where the target 3D CG objects were two cuboids of different heights and the physical projection surface was a corner-shaped screen.

object.

\subsection{Rendering pipeline}

We develop a new rendering pipeline for the projection images based on the conventional two-pass rendering technique [59]. The original algorithm consists of off-screen and on-screen rendering passes. It requires the positions of an observer and a projector as well as the shapes of the projection surfaces and the 3D CG object to be rendered. Next, (1) the off-screen rendering is performed to generate the desired appearance of the 3D object and its depth map for the observer's viewpoint. (2) The rendered image is then projected onto the surface from the observer's viewpoint in the virtual space. (3) After that, the on-screen rendering is performed, in which the newly textured surface is captured from the projector's viewpoint. (4) Finally, the captured image is projected in the physical space. We incorporate the depth filtering and lens breathing compensation into the original two-pass rendering algorithm.

In our pipeline, the depth filtering is performed in the first process of the original algorithm. In addition to the rendering of the 3D CG object and its depth map, we obtain the depth map of the projection surfaces for the observer's viewpoint (the first row of Fig. 6. Note that stereoscopic PM systems generally assume that the shape of a projection surface is obtained in advance and its pose is tracked online. We divide the rendered image into several parts using Eq. 3 such that each part is 
projected when the ETL's optical power becomes equal to a certain sampled value. As described in Sect. 3.1. the number of sampled optical powers is $N^{\prime}$, and, thus, we render $N^{\prime}$ images from the above process. We then apply depth filtering (Eq. 4p) to these images (the second row of Fig. 6). Next, the lens breathing compensation is performed in the second process of the original two-pass rendering algorithm, in which we resize the images that are rendered in the first process. We develop an efficient technique for the implementation of the resizing principle described in Sect. 3.3 In particular, we found that the resizing can be performed just by modifying the FOV of the virtual projector in the second process because (1) the resizing is performed with respect to the optical axis of the eye, and (2) the same resizing factor is applied to each divided part of the 3D CG object (Eq.5). Specifically, as shown in Fig.5 (.c2), the FOVs before and after modification are as follows:

$$
\theta_{p, f}=\arctan \frac{h_{p, f}}{d_{e}+d_{p}}, \theta_{p, f}^{\prime}=\arctan \frac{h_{p, f}^{\prime}}{d_{e}+d_{p}} .
$$

Therefore, we modify the FOV of the virtual projector by multiplying it by the factor of $\frac{\theta_{p, f}^{\prime}}{\theta_{p, f}}$ when we project the rendered image in the second process of our two-pass rendering pipeline. The third row of Fig. 6 shows an example of a rendered image after the lens breathing compensation has been applied.

\section{EXPERIMENT}

\subsection{Experimental setup}

We constructed a prototype system consisting of a pair of LC shutters, a pair of ETLs, and a synchronized high-speed projector (Fig. 11). We assembled our active-shutter glasses by attaching each ETL (Optotune AG, EL-16-40-TC, aperture: $16 \mathrm{~mm}$, optical power range: $-10 \mathrm{D}$ to $10 \mathrm{D})$ onto an LC shutter taken from off-the-shelf active-shutter glasses (RV-3DGDLP1, field rate: 96-144 Hz) and inserted a pair of the combined device into an eyeglass frame fabricated using an FDM $3 \mathrm{D}$ printer to form a wearable $(69 \times 128 \times 67 \mathrm{~mm}, 200 \mathrm{~g})$ device. The digital signal (sinusoidal wave) to control the ETLs was generated by a workstation (CPU: Intel Xeon E3-1225 v5 3.30GHz, RAM: 32GB, GPU: NVIDIA Quadro K620), input into a D/A converter (National Instruments, USB-6211), and converted into an analog voltage. This voltage was then converted to an electric current through a custom amplifier circuit using an op-amp (LM675T). Finally, the current was fed to the ETLs. The LC shutters were controlled using a circuit consisting of a motor driver (TOSHIBA, TA7291P). We employed an off-the-shelf high-speed projector (Inrevium, TB-UK-DYNAFLASH, $1024 \times 768$ pixels, 330 ANSI lumen) that can project 8 -bit grayscale images at 2,000 frames per second (fps) 1 The projection images were generated by the workstation and sent to the projector via a PCI Express interface. We manually adjusted the projector location so that the projected result always appeared focused on a projection surface. We manually measured the surface's shape and its pose relative to the ETLs offline. In Sect. 4.5. a surface was tracked using a motion capture system consisting of five cameras (NaturalPoint, OptiTrack Prime $17 \mathrm{~W}$ ). The shape and pose information was used to obtain the depth map in our rendering pipeline.

The workstation was able to generate a trigger signal for the projector and a control signal for the LC shutters along with the sinusoidal wave for the ETLs. However, the D/A converter only had two output channels, which was not sufficient for sending all the signals. Therefore, we used an additional microcontroller (Arduino Uno) to synchronize the display timing of each projection image and the opening/closing timing of the LC shutters. In our system, the workstation sent the sinusoidal wave $i[\phi]$ and a single global trigger signal at $\phi=0$ to the D/A converter. The trigger signal was then transferred to the microcontroller, which generated the trigger and the control signal using the delays of $\delta t_{p}$, $\delta t_{c 2 o}$, and $\delta t_{o 2 c}$, and sent to the projector and the controlling circuit of the LC shutters. The system architecture is depicted in Fig.7]

${ }^{1}$ Although the frame rate on the specification sheet is $1,000 \mathrm{fps}$, we experimentally discovered that the projector could project grayscale images at 2,000 fps.

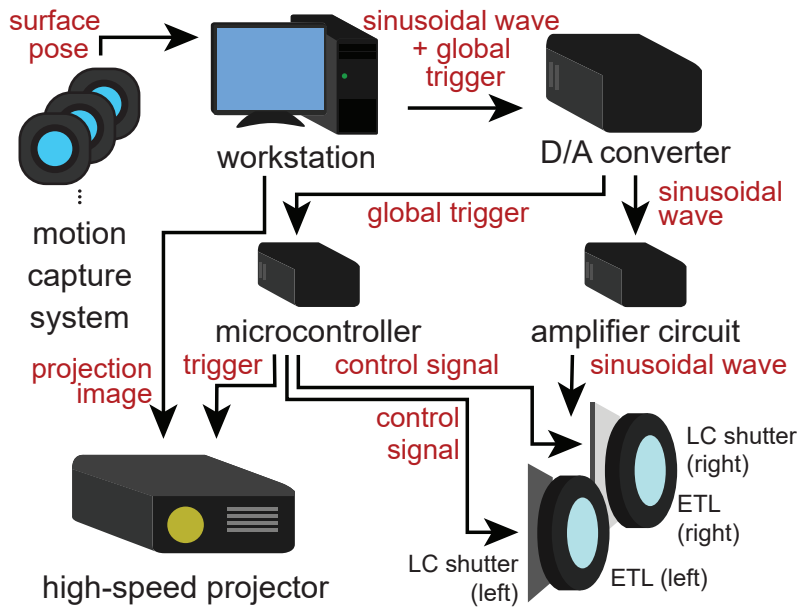

Fig. 7. System architecture.
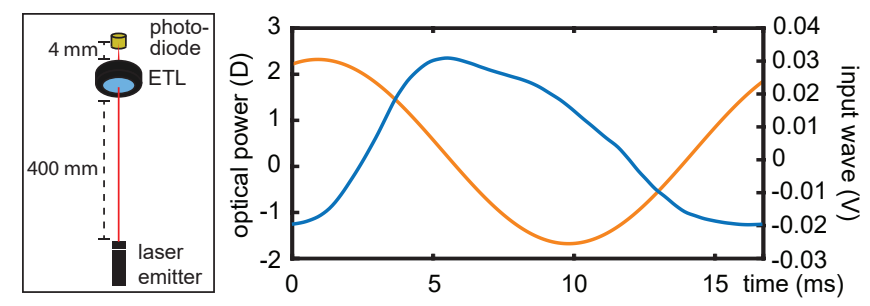

Fig. 8. Optical power measurement: (left) experimental setup, (right) one cycle of input sinusoidal wave (orange line), and the resultant optical power of an ETL (blue line).

\subsubsection{System calibration}

The system calibration was conducted as follows. We built an optical power measurement system using a photodiode and a laser emitter (wavelength: $635 \mathrm{~nm}$, output power: $0.9 \mathrm{~mW}$ ) (Fig. 8). We placed the ETL at about $400 \mathrm{~mm}$ away from the laser emitter. The photodiode was placed about $4 \mathrm{~mm}$ behind the ETL, and it measured the laser beam passing through the ETL. Raising the optical power of the ETL reduced the laser's spot size on the photodiode (i.e., increased the laser power density), enhancing the electric current created in the photodiode. We indirectly measured the ETL's optical power in this manner. For the calibration of the ETL, we input a sinusoidal wave $i[\phi]$ to the ETL and measured one cycle of its output optical power $v[\phi]$. The frequency of the wave was maintained at $60 \mathrm{~Hz}$ throughout the experiment, which was higher than the CFF. According to the discussion in Sect. 3.1 the sweep range was determined based on the desired location range of the virtual images (i.e., $d_{v, n}$ and $d_{v, f}$ ) on the nearest physical surface from the ETL. In the following experiments, except for the user study in Sect. 4.6. we considered the nearest distance as $500 \mathrm{~mm}$, and $d_{v, n}=$ $167 \mathrm{~mm}$ and $d_{v, f}=\infty \mathrm{mm}$. Therefore, the required sweep range was between $-1.0 \mathrm{D}$ and 2.0 D according to Eq. 1 . We can still modulate the location of the virtual image of a projected 3D CG object onto a surface whose distance from the ETL is shorter than $500 \mathrm{~mm}$. But in such a case, the modulation range becomes narrower than that between $d_{v, n}$ and $d_{v, f}$. We manually searched for the offset and amplitude of the input sinusoidal wave by which the output optical power of the ETL can cover the target range and found the suitable values (offset: $2.5 \mathrm{mV}$, amplitude: $28 \mathrm{mV}$ ). Figure 8 shows the measured optical power $v[\phi]$ by the input wave. From the figure, we found that the output values did not form a clean sinusoidal wave. Therefore, we recorded one cycle of the output wave along with the corresponding input wave and stored it on the microcontroller to be able to look up the optical power at a given phase of the input wave.

To synchronize the ETLs and the high-speed projector, we used the same photodiode to measure the delay for the high-speed projector between a trigger signal being sent from the microcontroller and the 


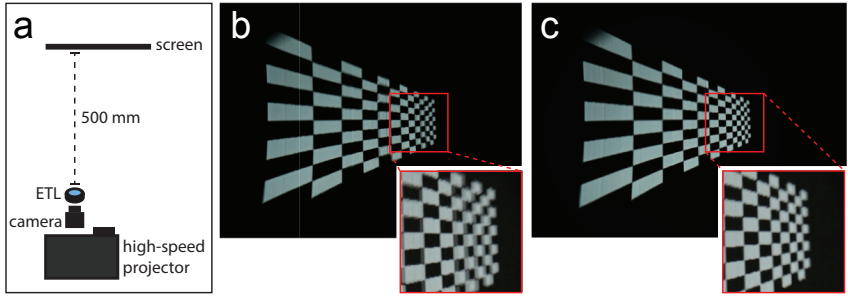

Fig. 9. Experimental validation of the proposed lens breathing compensation technique. The projected 3D CG object was a slanted plane with a checker pattern. (a) Experimental setup. The object's appearances without (b) and with (c) the compensation.

actual projection. As a result, we found that the delay was $0.15 \mathrm{~ms}$ $\left(=\delta t_{p}\right)$. We also measured the delay for the LC shutters and found that the closing transition took $0.1 \mathrm{~ms}\left(=\delta t_{o 2 c}\right)$, while the opening transition took $3.0 \mathrm{~ms}\left(=\delta t_{c 2 o}\right)$. This delay information was also stored in the microcontroller. The microcontroller received the global trigger signal, computed when the trigger and control signals should be sent to the projector and LC shutters based on the recorded optical power waveform and the delay information, and, finally, sent the signals to the devices.

\subsection{Lens breathing compensation}

We verified whether the proposed lens breathing compensation improved the gap or overlap between adjacent areas of a projected 3D CG object, which were observed with different optical powers. The ETL was attached to a camera (Sony $\alpha 7 \mathrm{~S}$ ) with a suitable lens (Sony SEL24F14GM, focal length: $24 \mathrm{~mm}$ ) that was used to capture the experimental results with a shutter speed of $1 / 60$ seconds. We placed a flat screen at $500 \mathrm{~mm}$ away from the ETL such that it was perpendicular to the ETL's optical axis. The displayed 3D CG object was a textured plane. The virtual image of the object was placed such that the distance of the left edge from the ETL was $333 \mathrm{~mm}$, and that from the right edge was $1666 \mathrm{~mm}$. The attached texture was a black and white checker pattern. We projected each part of the 3D CG object when the ETL's optical power was either $-1.0 \mathrm{D},-0.5 \mathrm{D}, 0.0 \mathrm{D}, 0.5 \mathrm{D}, 1.0 \mathrm{D}$, or $1.5 \mathrm{D}$. We captured the projected result under two conditions: when the lens breathing compensation was (1) applied and (2) not applied.

Figure 9 shows the results. Comparing the results of the two conditions, we found that the appearance of the checkerboard texture significantly improved when the proposed compensation method was applied. In particular, the unnatural overlaps of the textures degraded the image quality of the projected result without the compensation, while such artifacts were almost not visible in the result with the compensation. Therefore, we confirmed that the proposed lens breathing compensation technique works well to alleviate the artifacts caused by the lens breathing phenomenon.

\subsection{Multifocal appearance of a projected object on a flat static surface}

We validated our technique using a flat, static screen. As shown in Fig. 1 1 , we placed the screen $500 \mathrm{~mm}$ away from the ETL, which was attached to the same camera mentioned in Sect.4.2 In addition, three physical objects were placed at different distances from the ETL, which were $333 \mathrm{~mm}$ (near), $500 \mathrm{~mm}$ (middle), and 1,000 mm (far). We projected three Stanford bunnies on the screen such that the distance of the virtual image of each bunny from the ETL was the same as one of the physical objects. Specifically, the near, middle, and far bunnies were projected when the ETL's optical power was $-1.0 \mathrm{D}, 0.0 \mathrm{D}$, and 1.0 $\mathrm{D}$, respectively, which were computed using Eq. 1 The near, middle, and far bunnies were displayed from the bottom to top of the screen. The physical objects were illuminated by a uniform white image when the optical power was $0.0 \mathrm{D}$. We captured the scene using the camera by changing its focus distance from $333 \mathrm{~mm}$ to $1,000 \mathrm{~mm}$.

Figures 1 ,, $1 \mathrm{~d}$, and 1 e show the results. When the focus distance of the camera was $333 \mathrm{~mm}$, the physical object that was placed in the near position appeared more focused than the other physical objects.

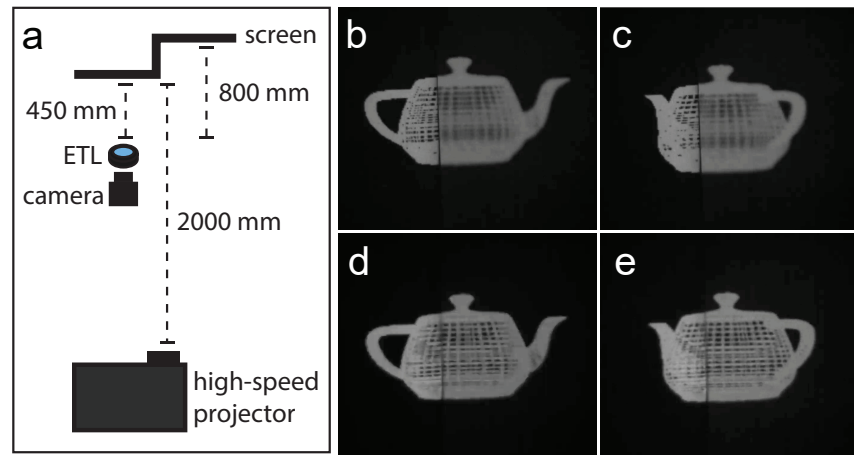

Fig. 10. Multifocal appearance of a rotating Utah teapot projected onto a step surface. (a) Experimental setup. (b, c) The projected object observed through the ETL with a fixed optical power of zero. (d, e) The projected object observed using the proposed method.

Similarly, the image of the bottom bunny, which was projected when the optical power was $-1.0 \mathrm{D}$, appeared more focused than that of the other projected bunnies. We obtained the same observations when the camera's focus distance was $500 \mathrm{~mm}$ and $1,000 \mathrm{~mm}$ as well. Therefore, we concluded that the proposed method could provide a multifocal view in PM while presenting the physical objects without any focus modulations.

\subsection{Multifocal appearance of a projected object on a non- planar surface}

A unique technical challenge when attempting to mitigate the VAC in stereoscopic PM is that the display surface can potentially be nonplanar. Thus, we verified whether the proposed system could provide the correct focus cues when the projection screen is non-planar. We placed a step surface such that the two planes were facing the ETL. The distance of the planes from the ETL were $450 \mathrm{~mm}$ and $800 \mathrm{~mm}$, respectively. We projected a Utah teapot such that it covered the two planes. We placed the projector about $2.0 \mathrm{~m}$ away from the surface, which caused the projected images to be focused on the both planes. Using our technique, the virtual image of the object was positioned such that the center of the object was at the same distance from the ETL as the front plane. We projected each part of the object when the ETL's optical power was either $-1.0 \mathrm{D},-0.5 \mathrm{D}, 0.0 \mathrm{D}$, or $0.5 \mathrm{D}$. As a baseline, we also captured the object while keeping the optical power fixed at $0.0 \mathrm{D}$. Therefore, there were two conditions for which the scene was recorded: (1) with and (2) without our technique.

Figure 10 shows the results captured by a camera (Ximea MQ013CG$\mathrm{ON}$ ) with a C-mount lens (focal length: $12 \mathrm{~mm}$ ) to which the ETL was attached. Comparing the results of the two conditions, we found that all parts of the teapot observed using our technique appeared focused when we adjusted the camera lens such that the focus point corresponds to the front projection plane. On the other hand, the right side of the teapot appeared blurred in the condition where our technique was not used. Thus, we concluded that the proposed technique could successfully realize multifocal PM for a non-planar screen.

\subsection{Multifocal appearance of a projected object on a mov- ing surface}

Another unique technical challenge when attempting to mitigate the VAC in stereoscopic PM is that the display surface can potentially move. Thus, we verified whether the proposed system could provide the correct focus cues when the projection screen moves in an experimental setup, shown in Fig. 11 A flat screen was manually moved between $300 \mathrm{~mm}$ and $500 \mathrm{~mm}$ from the same camera mentioned in Sect.4.4 The projected object was a Stanford bunny such that the 3D position of its virtual image in the scene was fixed regardless of the screen pose. We controlled the timing of the projection so that the bunny always appeared focused. The bunny image was projected when the ETL's optical power was either $-0.5 \mathrm{D}, 0.0 \mathrm{D}, 0.5 \mathrm{D}, 1.0 \mathrm{D}$, or $1.5 \mathrm{D}$. As a baseline, we also recorded a video of the bunny while keeping the 

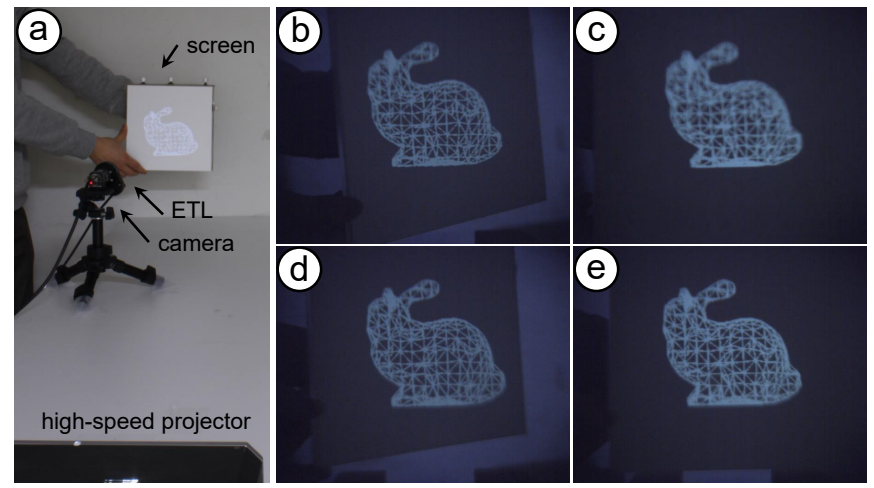

Fig. 11. Multifocal appearance of a Stanford bunny projected onto a flat surface freely moved by hand. (a) Experimental setup. (b, c) The projected object observed through the ETL with a fixed optical power of zero. (d, e) The projected object observed using the proposed method. Need revision

optical power fixed at $0.0 \mathrm{D}$. Therefore, the same two experimental conditions as discussed in Sect. 4.4 were established. As indicated in Sect. 4.1 the screen pose was tracked using a motion capture system.

Figure 11 shows the results that were captured at different screen poses. First, we ensured that the size and position of the bunny were consistent throughout the video-recording process in both experimental conditions. This indicates that our two-pass rendering pipeline worked correctly. Comparing the results of the two conditions, we found that the bunny observed using our technique always appeared focused, while the one projected without our technique appeared blurred at certain screen poses. Thus, we concluded that the proposed technique could successfully realize multifocal PM for a moving screen.

\subsection{User study of depth-matching}

We conducted a user study to investigate the effect of the VAC on the depth estimation accuracy of a human observer in stereoscopic PM Previous studies have revealed that the VAC caused distortions in the perceived depth; more specifically, people overestimated the depth of an AR target when using conventional OST displays [56, 66, 67]. Our user study investigated the depth estimation accuracy in a depth-matching experiment that was designed based on the previous studies. Each participant moved a physical pointer to match its depth with a virtual target displayed using stereoscopic PM. We prepared two experimental conditions. The first one was the "proposed" condition, where the VAC was mitigated by our system, and the second one was the "conventional" condition, where the VAC was not mitigated and only the binocular cue was correct. Based on the results of the previous studies [56 66, 67], the hypotheses of our study were formulated as follows:

H1: The depth was overestimated when the VAC was not mitigated in stereoscopic PM.

H2: The depth estimation was more accurate when the VAC was mitigated by our proposed system.

\subsubsection{Experimental setup and procedure}

We built an experimental system in a dark room, as shown in Fig. 12. Participants sat on a chair and placed their faces on a chin rest. We attached our active-shutter glasses consisting of the ETLs and the LC shutters to the chin rest such that the participants could see the experimental scene through our glasses. The virtual target was a checker pattern, which was projected onto a flat screen placed $2.5 \mathrm{~m}$ away from the ETLs. The visual angle of the target was fixed at 2.4 degrees throughout the experiment. By providing the appropriate disparities to both eyes, our system presented the virtual target at five distances from the ETLs: $300 \mathrm{~mm}, 364 \mathrm{~mm}, 400 \mathrm{~mm}, 444 \mathrm{~mm}$, and $500 \mathrm{~mm}$. The sweep range of the ETL's optical power in this experiment was $-2.6 \mathrm{D}$ to $0.0 \mathrm{D}$. Under the proposed condition, the virtual target was projected when the ETL's optical power was $-2.6 \mathrm{D},-2.1 \mathrm{D}$, and -1.6 D for the
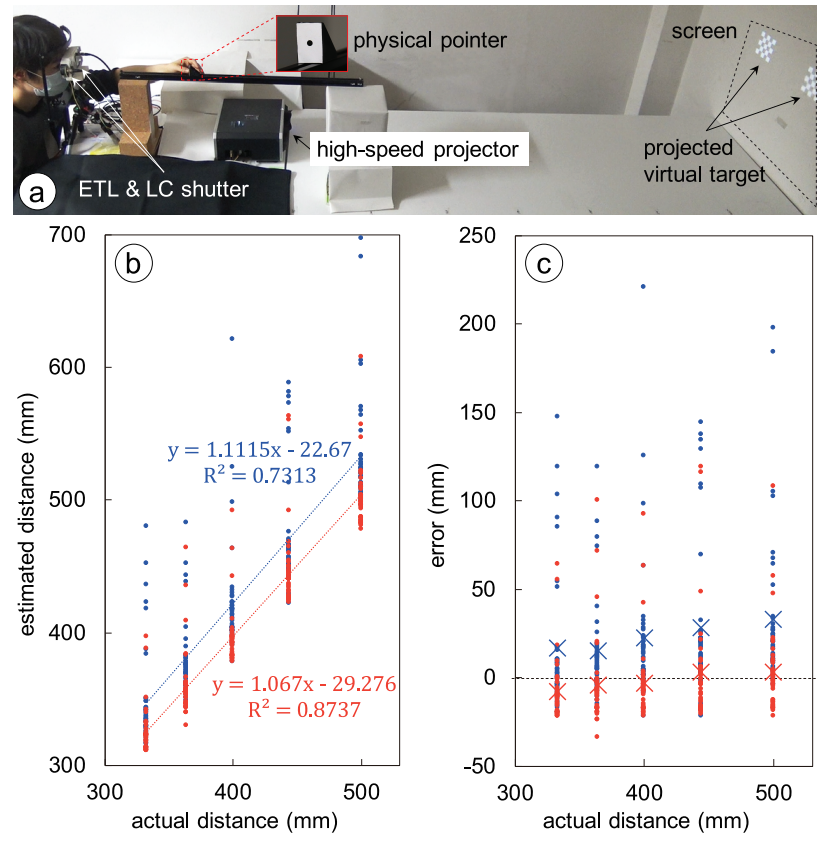

Fig. 12. Depth-matching experiment. (a) Experimental setup where a participant manually moved a physical pointer to match its depth with a projected virtual target (checker pattern). Note that the study was conducted under a dark environment. (b) The scatter plot of the estimated against the actual distance with fitted linear equations. The red and blue plots represent the proposed and conventional conditions, respectively. (c) Errors of the estimated from the actual distance in both conditions; " $x$ " indicates the mean value.

target distances of $300 \mathrm{~mm}, 400 \mathrm{~mm}$, and $500 \mathrm{~mm}$, respectively. We applied the depth filtering technique to present the focus cues for the other two target distances. For the conventional condition, the virtual target was projected when the optical power was zero for all the target distances. For both conditions, the physical pointer was attached to a linear slider using which a participant could move the pointer along the direction of the ETL's optical axis. The system illuminated the physical pointer when the ETL's optical power was zero using a synchronized light.

We recruited ten participants from a local university. All participants were naïve to the purpose of the experiment and had normal or corrected-to-normal vision. We divided the participants into two groups; half of them performed depth-matching tasks under the proposed condition on the first day and did the same under the conventional condition on the second day, while the other half performed the tasks in the opposite order. In a preparation block, we measured the interpupillary distance (IPD) for each participant and accordingly adjusted the distance between the two ETLs. Then, we asked them to adjust their head positions by observing a calibration pattern to ensure that the disparities of the physical pointer and the virtual target were identical at the same distance. Specifically, we projected a cross pattern onto the screen for each eye and positioned a physical object on which the same cross pattern was printed such that all the crosses corresponded to the participant's retina when their head position was correct. After the preparation block, each participant performed the following depthmatching tasks, including a training task. In each task, they were asked to move the physical pointer by hand until they perceived that it was located at the same depth as the virtual target. We prepared five depths as described above, and each participant performed the depth-matching tasks four times for each depth. Thus, there were 20 (=5 depth $\times 4$ repetitions) trials in addition to the training trial for each condition. The order of the trials was counterbalanced among the participants. Further, the participants were allowed to take as much time as they needed to complete each task. We told them that they could give up on a task if they could not perform the binocular fusion. After all the tasks were 
completed, we asked each participant whether they perceived the flicker of the displayed object.

\subsubsection{Result}

All the participants were able to perform the binocular fusion in all the tasks and reported that they did not perceive any flickers during the experiment. Figure $12 \mathrm{p}$ shows the results plotted as a scatter plot of the estimated against the actual distance. Following previous studies $\mid 56$ 66. 67], we calculated the linear functions that predict the estimated distance from the actual distance and used multiple regression methods to determine whether the slopes and intercepts significantly differed between the conditions. While Fig. 12 p shows all 400 data points $(=20$ trials $\times 2$ conditions $\times 10$ participants), we averaged the responses for the four repetitions for each participant, which reduced the size of the dataset to 100 points for the multiple regression analysis [67] We then compared the two linear fits of the proposed and conventional conditions. Our multiple regression analysis found that the slopes of the linear fits did not significantly differ $\left(F_{1,96}=0.218, p=0.64\right)$, while the intercepts did $\left(F_{1,97}=19.552, p<0.01\right)$.

Figure 12, illustrates the errors of the estimated distance from the actual distance, where positive errors indicate the overestimation. The participants overestimated the depth by $22.86 \mathrm{~mm}$ on average in the conventional condition, while the error was much smaller $(-1.93 \mathrm{~mm})$ in the proposed condition. We performed a two-way analysis of variance (ANOVA) for the actual distance of the virtual target and the experimental condition to evaluate the estimation errors. Again, we averaged the responses of the four repetitions for each participant. The ANOVA showed the main effect of the experimental condition $\left(F_{1,90}=18.234, p<0.01\right)$, but it did not show the main effect of the actual distance of the virtual target $\left(F_{4,90}=0.861, p=0.49\right)$ and the interaction effect $\left(F_{4,90}=0.088, p=0.99\right)$. Next, a post-hoc analysis was performed using Tukey's HSD method for pairwise comparison. The result showed a statistically significant difference between the proposed and conventional conditions $(p<0.01)$.

Therefore, both our hypotheses were supported by the results. In stereoscopic PM, the participants overestimated the depth of the virtual target when the VAC was not mitigated (H1) and estimated it more accurately when it was mitigated (H2). These trends are consistent with the previous results of OST-AR displays [56 66, 67].

\section{Discussion}

From the experimental results, we confirmed that our multifocal stereoscopic PM technique successfully worked for different types of projection screens. Specifically, it could provide the correct focus cues for static (Sect.4.3), non-planar (Sect.4.4, and moving surfaces (Sect.4.5. We also confirmed that our lens breathing compensation technique can be used to significantly reduce the associated artifacts (Sect.4.2). Further, because it is a general solution, it can be applied to other AR/VR multifocal and varifocal displays and improve their image qualities. Furthermore, the user study showed that the proposed technique could mitigate the VAC and significantly reduced the distortions in perceived depth in stereoscopic PM (Sect.4.6.

\subsection{Limitations}

Although we achieved the goal of this research, our technique still suffers from several technical limitations. First, the current system works properly only in a dark environment because a bright environment increases the black offset of a projected result and, consequently, degrades its contrast. Other PM systems also suffer from the same limitation because the environment lighting decreases the contrast of the projected imagery [7]. Previous attempts have addressed the limitation by illuminating the entire scene surface using multiple projectors, which can theoretically reproduce the original environment lighting [33]. In addition, by using large-aperture optics [22], the environment lighting can be naturally reproduced. A potential extension of our system is to apply anisotropic diffusers or retro-reflectors as projection screens [19 77], but this, on the other hand, limits the scalability of the system.

Second, the optical power of the ETL changes during each projection frame displayed because it is continuously modulated over time.
In addition, a certain amount of time is required for the projection of a 3D CG object to provide sufficient light intensity for an observer. As a result, an observer perceives the integration of the undesirable appearances of a projected image. Other multifocal displays that employ the focal sweep approach (see Sect. 2.2 suffer from the same limitation. Using a custom high-speed projector, we can illuminate the scene for a shorter duration to mitigate the image quality degradation, but this results in the image having a darker appearance. Another and more promising solution for overcoming this limitation is to apply a waveform optimization of ETL's drive signal [28], which will improve the image quality by maintaining the optical power at the desired values for a sufficient amount of time.

Third, as an implementation limitation, our system requires a highspeed projector. Although such a projector can currently be considered special equipment, the one we used in this paper is an off-the-shelf projector that is available in the market [73]. In addition, researchers have found in the last few years that a high-speed projector is essential for realizing immersive dynamic PM applications [20 53 69|. Although our current system applies a gray-scale projector, the manufacturer of the projector just launched a full-color version. Given this technical trend, high-speed projectors will probably soon become normal equipment in PM applications.

Foruth, because a projector generally suffers from a narrow depth of field (DOF), a part of the image projected on a non-planar surface does not appear focused in some cases. Here, even if the proposed technique successfully displays the virtual image of a projected object at the desired distance, the virtual image does not appear focused. This can be solved by attaching another ETL to the projector and applying a focal sweep to it |29.76|.

Last, the ETL significantly reduces the angle of viewing for an observer. This problem can be also seen in previous AR/VR displays that employ ETLs [10 36]. Considering the trend of the increasing aperture size of ETLs due to the constant innovation of manufacturers, we believe that this limitation will be overcome in the near future.

In summary, although there are currently several technical limitations that are common to the related fields, we believe that they do not immediately degrade the applicability of the proposed method.

\section{Conclusion}

This paper aimed at achieving multifocal stereoscopic PM to improve the mismatch between vergence and accommodation. To achieve this objective, we jointly designed the optics, hardware, and computational algorithms. Specifically, we attached ETLs to the LC shutters of activeshutter glasses to control both vergence and accommodation and synchronized them using an off-the-shelf high-speed projector. We applied a fast focal sweep to the ETLs and projected a 3D CG object from the projector onto physical surfaces when the distance of its virtual image was located exactly at a desired distance from the ETLs. Further, we solved three technical issues that are unique to the stereoscopic PM and developed a simple lens breathing compensation technique that can be applied to other multifocal and varifocal AR/VR displays. Using a proof-of-concept prototype, we have demonstrated that our technique can provide the correct focus cues required for different types of projection screens. We have also confirmed that the lens breathing compensation can eliminate artifacts such as overlaps between the adjacent areas of projected imagery. Finally, we validated the advantage provided by our technique with regard to the mitigation of the VAC by comparing it with conventional stereoscopic PM using a user study of a depth-matching task. A new branch of research associated with AR/VR displays has recently been enabled by the combination of projectors and near-eye optics [27 70,71], and this work can be categorized with the same. We will continue to investigate the possibility and applicability of this emerging framework.

\section{ACKNOWLEDGMENTS}

This work was supported by JSPS KAKENHI Grant Numbers JP18K19817 and JP20H05958 and JST, PRESTO Grant Number JPMJPR19J2, Japan. 


\section{REFERENCES}

[1] K. Akșit, W. Lopes, J. Kim, P. Shirley, and D. Luebke. Near-eye varifocal augmented reality display using see-through screens. ACM Trans. Graph., 36(6), Nov. 2017.

[2] K. Akşit, A. H. G. Niaki, E. Ulusoy, and H. Urey. Super stereoscopy technique for comfortable and realistic 3d displays. Opt. Lett., 39(24):69036906, Dec 2014.

[3] K. Akeley, S. J. Watt, A. R. Girshick, and M. S. Banks. A stereo display prototype with multiple focal distances. ACM Trans. Graph. 23(3):804-813, Aug. 2004.

[4] U. Y. Başak, S. Kazempourradi, C. Yilmaz, E. Ulusoy, and H. Urey. Dual focal plane augmented reality interactive display with gaze-tracker. $O S A$ Continuum, 2(5):1734-1745, May 2019.

[5] H. Benko, R. Jota, and A. Wilson. Miragetable: Freehand interaction on a projected augmented reality tabletop. In Proceedings of the SIGCHI Conference on Human Factors in Computing Systems, CHI '12, p. 199-208, 2012.

[6] H. Benko, A. D. Wilson, and F. Zannier. Dyadic projected spatial augmented reality. In Proceedings of the 27th Annual ACM Symposium on User Interface Software and Technology, UIST '14, p. 645-655, 2014.

[7] O. Bimber and R. Raskar. Spatial Augmented Reality: Merging Real and Virtual Worlds. A. K. Peters, Ltd., 2005.

[8] O. Bimber, G. Wetzstein, A. Emmerling, and C. Nitschke. Enabling viewdependent stereoscopic projection in real environments. In Fourth IEEE and ACM International Symposium on Mixed and Augmented Reality (ISMAR'05), pp. 14-23, 2005.

[9] J. Byun, S. Chae, Y. Yang, and T. Han. AIR: Anywhere Immersive Reality with User-Perspective Projection. In A. Peytavie and C. Bosch, eds., EG 2017 - Short Papers, 2017.

[10] P. Chakravarthula, D. Dunn, K. Akşit, and H. Fuchs. Focusar: Auto-focus augmented reality eyeglasses for both real world and virtual imagery. IEEE Transactions on Visualization and Computer Graphics, 24(11):2906-2916, 2018.

[11] P. Chakravarthula, Y. Peng, J. Kollin, H. Fuchs, and F. Heide. Wirtinger holography for near-eye displays. ACM Trans. Graph., 38(6), Nov. 2019.

[12] P. Chakravarthula, E. Tseng, T. Srivastava, H. Fuchs, and F. Heide. Learned hardware-in-the-loop phase retrieval for holographic near-eye displays ACM Trans. Graph., 39(6), Nov. 2020.

[13] J.-H. R. Chang, B. V. K. V. Kumar, and A. C. Sankaranarayanan. Towards multifocal displays with dense focal stacks. ACM Trans. Graph., 37(6), Dec. 2018.

[14] J.-H. R. Chang, A. Levin, B. V. K. V. Kumar, and A. C. Sankaranarayanan. Towards occlusion-aware multifocal displays. ACM Trans. Graph., 39(4), July 2020

[15] H.-S. Chen, Y.-J. Wang, P.-J. Chen, and Y.-H. Lin. Electrically adjustable location of a projected image in augmented reality via a liquid-crystal lens. Opt. Express, 23(22):28154-28162, Nov 2015.

[16] Q. Chen, Z. Peng, Y. Li, S. Liu, P. Zhou, J. Gu, J. Lu, L. Yao, M. Wang, and Y. Su. Multi-plane augmented reality display based on cholesteric liquid crystal reflective films. Opt. Express, 27(9):12039-12047, Apr 2019.

[17] S. A. Cholewiak, G. D. Love, P. P. Srinivasan, R. Ng, and M. S. Banks. Chromablur: Rendering chromatic eye aberration improves accommodation and realism. ACM Trans. Graph., 36(6), Nov. 2017.

[18] D. Dunn, C. Tippets, K. Torell, P. Kellnhofer, K. Akşit, P. Didyk, K. Myszkowski, D. Luebke, and H. Fuchs. Wide field of view varifocal near-eye display using see-through deformable membrane mirrors. IEEE Transactions on Visualization and Computer Graphics, 23(4):1322-1331, 2017.

[19] O. Eldes, K. Akşit, and H. Urey. Multi-view autostereoscopic projection display using rotating screen. Opt. Express, 21(23):29043-29054, Nov 2013

[20] K. Fukamizu, L. Miyashita, and M. Ishikawa. Elamorph projection: Deformation of $3 \mathrm{~d}$ shape by dynamic projection mapping. In 2020 IEEE International Symposium on Mixed and Augmented Reality (ISMAR), pp. 164-173, 2020.

[21] F. Heinrich, K. Bornemann, K. Lawonn, and C. Hansen. Depth perception in projective augmented reality: An evaluation of advanced visualization techniques. In 25th ACM Symposium on Virtual Reality Software and Technology, VRST'19, 2019.

[22] K. Hiratani, D. Iwai, P. Punpongsanon, and K. Sato. Shadowless projector: Suppressing shadows in projection mapping with micro mirror array plate.
In 2019 IEEE Conference on Virtual Reality and 3D User Interfaces (VR), pp. 1309-1310, 2019.

[23] M. Hirsch, G. Wetzstein, and R. Raskar. A compressive light field projection system. ACM Trans. Graph., 33(4), July 2014.

[24] D. M. Hoffman, A. R. Girshick, K. Akeley, and M. S. Banks. Vergenceaccommodation conflicts hinder visual performance and cause visual fatigue. Journal of Vision, 8(3):33-33, Mar 2008.

[25] X. Hu and H. Hua. Design and assessment of a depth-fused multi-focalplane display prototype. Journal of Display Technology, 10(4):308-316, 2014.

[26] F.-C. Huang, K. Chen, and G. Wetzstein. The light field stereoscope: Immersive computer graphics via factored near-eye light field displays with focus cues. ACM Trans. Graph., 34(4), July 2015.

[27] Y. Itoh, T. Kaminokado, and K. Akşit. Beaming displays. IEEE Transactions on Visualization and Computer Graphics, 27(5):2659-2668, 2021.

[28] D. Iwai, H. Izawa, K. Kashima, T. Ueda, and K. Sato. Speeded-up focus control of electrically tunable lens by sparse optimization. Scientific Reports, 9(1):12365, Aug 2019.

[29] D. Iwai, S. Mihara, and K. Sato. Extended depth-of-field projector by fast focal sweep projection. IEEE Transactions on Visualization and Computer Graphics, 21(4):462-470, 2015.

[30] C. Jang, K. Bang, G. Li, and B. Lee. Holographic near-eye display with expanded eye-box. ACM Trans. Graph., 37(6), Dec. 2018.

[31] Y. Jo, S. Lee, D. Yoo, S. Choi, D. Kim, and B. Lee. Tomographic projector: Large scale volumetric display with uniform viewing experiences. $A C M$ Trans. Graph., 38(6), Nov. 2019.

[32] P. V. Johnson, J. A. Parnell, J. Kim, C. D. Saunter, G. D. Love, and M. S. Banks. Dynamic lens and monovision 3d displays to improve viewer comfort. Opt. Express, 24(11):11808-11827, May 2016.

[33] B. Jones, R. Sodhi, M. Murdock, R. Mehra, H. Benko, A. Wilson, E. Ofek, B. MacIntyre, N. Raghuvanshi, and L. Shapira. Roomalive: Magical experiences enabled by scalable, adaptive projector-camera units. In Proceedings of the 27th Annual ACM Symposium on User Interface Software and Technology, p. 637-644, 2014.

[34] T. Kanamori, D. Iwai, and K. Sato. Pseudo-shape sensation by stereoscopic projection mapping. IEEE Access, 6:40649-40655, 2018.

[35] D. Kondo, R. Kijima, and Y. Takahashi. Dynamic anatomical model for medical education using free form projection display. In Proceedings of the 13th International Conference on Virtual Systems and Multimedia (VSMM), 2007

[36] R. Konrad, E. A. Cooper, and G. Wetzstein. Novel optical configurations for virtual reality: Evaluating user preference and performance with focustunable and monovision near-eye displays. In Proceedings of the 2016 CHI Conference on Human Factors in Computing Systems, p. 1211-1220, 2016.

[37] R. Konrad, N. Padmanaban, K. Molner, E. A. Cooper, and G. Wetzstein. Accommodation-invariant computational near-eye displays. ACM Trans. Graph., 36(4), July 2017.

[38] G.-A. Koulieris, B. Bui, M. S. Banks, and G. Drettakis. Accommodation and comfort in head-mounted displays. ACM Trans. Graph., 36(4), July 2017.

[39] D. Lanman and D. Luebke. Near-eye light field displays. ACM Trans. Graph., 32(6), Nov. 2013.

[40] D. Lindlbauer, J. E. Grønbæk, M. Birk, K. Halskov, M. Alexa, and J. Müller. Combining shape-changing interfaces and spatial augmented reality enables extended object appearance. In Proceedings of the 2016 CHI Conference on Human Factors in Computing Systems, p. 791-802, 2016.

[41] S. Liu, Y. Li, P. Zhou, Q. Chen, S. Li, Y. Liu, Y. Wang, and Y. Su. Full-color multi-plane optical see-through head-mounted display for augmented reality applications. Journal of the Society for Information Display, 26(12):687-693, 2018.

[42] P. Llull, N. Bedard, W. Wu, I. Tošić, K. Berkner, and N. Balram. Design and optimization of a near-eye multifocal display system for augmented reality. In Imaging and Applied Optics 2015, p. JTh3A.5. Optical Society of America, 2015.

[43] G. D. Love, D. M. Hoffman, P. J. Hands, J. Gao, A. K. Kirby, and M. S. Banks. High-speed switchable lens enables the development of a volumetric stereoscopic display. Opt. Express, 17(18):15716-15725, Aug 2009.

[44] C. Lu, P. Chakravarthula, Y. Tao, S. Chen, and H. Fuchs. Improved vergence and accommodation via purkinje image tracking with multiple cameras for ar glasses. In 2020 IEEE International Symposium on Mixed 
and Augmented Reality (ISMAR), pp. 320-331, 2020.

[45] K. J. MacKenzie, D. M. Hoffman, and S. J. Watt. Accommodation to multiple-focal-plane displays: Implications for improving stereoscopic displays and for accommodation control. Journal of Vision, 10(8):22-22, Jul 2010.

[46] A. Maimone and H. Fuchs. Computational augmented reality eyeglasses. In IEEE International Symposium on Mixed and Augmented Reality (ISMAR), pp. 29-38, 2013.

[47] A. Maimone, A. Georgiou, and J. S. Kollin. Holographic near-eye displays for virtual and augmented reality. ACM Trans. Graph., 36(4), July 2017.

[48] A. Maimone, D. Lanman, K. Rathinavel, K. Keller, D. Luebke, and H. Fuchs. Pinlight displays: Wide field of view augmented reality eyeglasses using defocused point light sources. ACM Trans. Graph., 33(4), July 2014.

[49] A. Maimone and J. Wang. Holographie optics for thin and lightweight virtual reality. ACM Trans. Graph., 39(4), July 2020.

[50] N. Matsuda, A. Fix, and D. Lanman. Focal surface displays. ACM Trans. Graph., 36(4), July 2017.

[51] O. Mercier, Y. Sulai, K. Mackenzie, M. Zannoli, J. Hillis, D. Nowrouzezahrai, and D. Lanman. Fast gaze-contingent optimal decompositions for multifocal displays. ACM Trans. Graph., 36(6), Nov. 2017.

[52] R. Narain, R. A. Albert, A. Bulbul, G. J. Ward, M. S. Banks, and J. F. O'Brien. Optimal presentation of imagery with focus cues on multi-plane displays. ACM Trans. Graph., 34(4), July 2015.

[53] T. Nomoto, W. Li, H.-L. Peng, and Y. Watanabe. Dynamic projection mapping with networked multi-projectors based on pixel-parallel intensity control. In SIGGRAPH Asia 2020 Emerging Technologies, 2020.

[54] N. Okutani, T. Takezawa, D. Iwai, and K. Sato. Stereoscopic capture in projection mapping. IEEE Access, 6:65894-65900, 2018.

[55] N. Padmanaban, R. Konrad, T. Stramer, E. A. Cooper, and G. Wetzstein. Optimizing virtual reality for all users through gaze-contingent and adaptive focus displays. Proceedings of the National Academy of Sciences, 114(9):2183-2188, 2017.

[56] E. Peillard, Y. Itoh, G. Moreau, J. M. Normand, A. Lécuyer, and F. Argelaguet. Can retinal projection displays improve spatial perception in augmented reality? In IEEE International Symposium on Mixed and Augmented Reality (ISMAR), pp. 80-89, 2020.

[57] T. Pejsa, J. Kantor, H. Benko, E. Ofek, and A. Wilson. Room2room: Enabling life-size telepresence in a projected augmented reality environment. In Proceedings of the 19th ACM Conference on Computer-Supported Cooperative Work and Social Computing, CSCW '16, p. 1716-1725, 2016.

[58] Y. Peng, S. Choi, N. Padmanaban, and G. Wetzstein. Neural holography with camera-in-the-loop training. ACM Trans. Graph., 39(6), Nov. 2020.

[59] R. Raskar, G. Welch, M. Cutts, A. Lake, L. Stesin, and H. Fuchs. The office of the future: A unified approach to image-based modeling and spatially immersive displays. In Proceedings of the 25th Annual Conference on Computer Graphics and Interactive Techniques, SIGGRAPH '98, p. 179-188, 1998.

[60] K. Rathinavel, H. Wang, A. Blate, and H. Fuchs. An extended depth-atfield volumetric near-eye augmented reality display. IEEE Transactions on Visualization and Computer Graphics, 24(11):2857-2866, 2018.

[61] J. P. Rolland, M. W. Krueger, and A. Goon. Multifocal planes headmounted displays. Appl. Opt., 39(19):3209-3215, Jul 2000.

[62] S. Schmidt, G. Bruder, and F. Steinicke. Moving Towards Consistent Depth Perception in Stereoscopic Projection-based Augmented Reality. In ICAT-EGVE 2017 - International Conference on Artificial Reality and Telexistence and Eurographics Symposium on Virtual Environments, 2017.

[63] S. Schmidt, G. Bruder, and F. Steinicke. Effects of virtual agent and object representation on experiencing exhibited artifacts. Computers \& Graphics, 83:1-10, 2019.

[64] S. Schmidt, G. Bruder, and F. Steinicke. Depth perception and manipulation in projection-based spatial augmented reality. PRESENCE: Virtual and Augmented Reality, 27(2):242-256, 2020.

[65] Sheng Liu, Dewen Cheng, and Hong Hua. An optical see-through head mounted display with addressable focal planes. In 2008 7th IEEE/ACM International Symposium on Mixed and Augmented Reality, pp. 33-42, 2008.

[66] G. Singh, S. R. Ellis, and J. E. Swan. The effect of focal distance, age, and brightness on near-field augmented reality depth matching. IEEE Transactions on Visualization and Computer Graphics, 26(2):1385-1398, 2020.

[67] J. E. Swan, G. Singh, and S. R. Ellis. Matching and reaching depth judgments with real and augmented reality targets. IEEE Transactions on Visualization and Computer Graphics, 21(11):1289-1298, 2015.

[68] T. Takezawa, D. Iwai, K. Sato, T. Hara, Y. Takeda, and K. Murase. Material surface reproduction and perceptual deformation with projection mapping for car interior design. In 2019 IEEE Conference on Virtual Reality and $3 D$ User Interfaces (VR), pp. 251-258, 2019.

[69] D. Tone, D. Iwai, S. Hiura, and K. Sato. Fibar: Embedding optical fibers in $3 \mathrm{~d}$ printed objects for active markers in dynamic projection mapping. IEEE Transactions on Visualization and Computer Graphics, 26(5):2030-2040, 2020.

[70] T. Ueda, D. Iwai, T. Hiraki, and K. Sato. Illuminated focus: Vision augmentation using spatial defocusing via focal sweep eyeglasses and high-speed projector. IEEE Transactions on Visualization and Computer Graphics, 26(5):2051-2061, 2020.

[71] T. Ueda, D. Iwai, and K. Sato. Illuminatedzoom: spatially varying magnified vision using periodically zooming eyeglasses and a high-speed projector. Opt. Express, 29(11):16377-16395, May 2021.

[72] J. P. Wann, S. Rushton, and M. Mon-Williams. Natural problems for stereoscopic depth perception in virtual environments. Vision Research, 35(19):2731 - 2736, 1995.

[73] Y. Watanabe, G. Narita, S. Tatsuno, T. Yuasa, K. Sumino, and M. Ishikawa. High-speed 8-bit image projector at 1,000 fps with $3 \mathrm{~ms}$ delay. In Proceedings of International Display Workshops, pp. 1064-1065, 2015.

[74] S. J. Watt, K. J. MacKenzie, and L. Ryan. Real-world stereoscopic performance in multiple-focal-plane displays: How far apart should the image planes be? In A. J. Woods, N. S. Holliman, and G. E. Favalora, eds., Stereoscopic Displays and Applications XXIII, vol. 8288, pp. 474 - 484. International Society for Optics and Photonics, SPIE, 2012.

[75] X. Xia, Y. Guan, A. State, P. Chakravarthula, K. Rathinavel, T. J. Cham, and H. Fuchs. Towards a switchable ar/vr near-eye display with accommodation-vergence and eyeglass prescription support. IEEE Transactions on Visualization and Computer Graphics, 25(11):3114-3124, 2019.

[76] H. Xu, L. Wang, S. Tabata, Y. Watanabe, and M. Ishikawa. An extended depth-of-field projection method using a high-speed projector with a synchronized oscillating variable focus lens. In J.-H. Lee, Q.-H. Wang, and T.-H. Yoon, eds., Advances in Display Technologies X, vol. 11304, pp. 97 103. International Society for Optics and Photonics, SPIE, 2020.

[77] T. Yoshida, S. Kamuro, K. Minamizawa, H. Nii, and S. Tachi. Repro3d: Full-parallax $3 \mathrm{~d}$ display using retro-reflective projection technology. In ACM SIGGRAPH 2010 Emerging Technologies, 2010.

[78] T. Zhan, J. Xiong, J. Zou, and S.-T. Wu. Multifocal displays: review and prospect. PhotoniX, 1(1):10, Mar 2020. 